\title{
Cardiotrophin-1 and leptin as cardiovascular risk markers in male patients with obstructive sleep apnea syndrome
}

Isil Cakir ${ }^{1}$, Mustafa Uluhan ${ }^{2}$

${ }^{1}$ Department of Clinical Biochemistry, Kayseri Education and Research Hospital, Kayseri, Turkey

${ }^{2}$ Clinics of Chest Diseases, Kayseri Education and Research Hospital, Kayseri, Turkey

Submitted: 16 April 2018

Accepted: 1 August 2018

Arch Med Sci Atheroscler Dis 2018; 3: e123-e128

DOI: https://doi.org/10.5114/amsad.2018.79407

Copyright $\odot 2018$ Termedia \& Banach

\section{Abstract}

Introduction: Elevated cardiotrophin-1 (CT-1) and leptin levels are important risk factors for cardiovascular diseases (CVDs). Obstructive sleep apnea syndrome (OSAS) has also been reported to increase this risk. The aim of this study is to evaluate serum concentrations of CT-1 and leptin in patients with OSAS and whether there is a possible association between CT-1, leptin and OSAS severity.

Material and methods: Fifty newly diagnosed OSAS patients and thirty nonapneic snoring subjects participated in this study. Fasting serum lipid profile markers were evaluated. The measurements of serum CT-1 and leptin levels were carried out using human ELISA kits.

Results: Significant differences were found in the serum CT-1 and leptin levels between the two groups. Serum median CT-1 levels in patient and control groups, respectively, were $19.47 ; 8.23 \mathrm{pg} / \mathrm{ml}$ and leptin levels were $2.07 ; 1.29 \mathrm{ng} / \mathrm{ml}(p<0.001)$. In the severe patient group, serum median CT-1 level was statistically significantly higher than the median level in the mild/ moderate group. There was no correlation between patients' leptin and lipid profile parameters and CT-1 concentrations were not associated with triglyceride, cholesterol or LDL cholesterol levels except HDL cholesterol: CT-1 levels were positively correlated with HDL levels $(p=0.02)$.

Conclusions: Both CT-1 and leptin were significantly elevated in the patient group. Furthermore, CT-1 and leptin were associated with OSAS and CT-1 was associated with the disease severity.

Key words: cardiotrophin-1, cardiovascular disease, leptin, obstructive sleep apnea syndrome.

\section{Introduction}

Obstructive sleep apnea syndrome (OSAS) is a widespread disorder with a prevalence of $2 \%$ and $4 \%$ of women and men, respectively. The male:female ratio of OSAS is approximately $3: 1$ [1]. The OSAS is a complicated chronic disease which can be diagnosed by the frequency of apnea and hypopnea while performing polysomnography (PSG) and treated by continuous positive airway pressure (CPAP), oral orthodontic treatment, medications, etc. Performing polysomnography is considered to be the gold standard to diagnose the disease characterized by obstruction of the upper airway that can be followed by anoxia, snoring and daytime sleepiness [2]. The severity of OSAS is classified as the

\author{
Corresponding author: \\ Isil Cakir \\ Department of \\ Clinical Biochemistry \\ Kayseri Education and \\ Research Hospital \\ 38010 Kayseri, Turkey \\ Phone: +90 3523368888 \\ E-mail: isilscakir@gmail.com
}


apnea-hypopnea index (AHI), which is an indicator of apnea-hypopnea episodes per hour during sleep [3]. Obstructive sleep apnea syndrome (OSAS) has been reported to lead to metabolic abnormalities and increase the risk of cardiovascular diseases (CVDs) such as myocardial infarction, atrial fibrillation, congestive heart failure, cardiac sudden death or only systemic hypertension. Except systemic hypertension, the relation between OSAS and CVDs remains unclear. Intermittent hypoxemia secondary to repeated episodes of apnea/ hypopnea may increase sympathetic activity. Sympathetic hyperactivation induces acute hemodynamic changes such as impairment of vasomotor reactivity, vascular inflammation, endothelial dysfunction, oxidative stress and metabolic disorders, and they all lead to complications during sleep in patients with OSAS [4-7]. Untreated OSAS may increase the incidence of non-fatal cardiovascular events, myocardial infarction and stroke. Despite all these explanations, the mechanisms how OSAS induces CVDs have not yet been fully elucidated.

Leptin, a $16 \mathrm{kDa}$ protein, was discovered by Zhang et al. in 1994. Leptin is an adipose-tissue specific adipokine, a member of the interleukin- 6 (IL-6) superfamily and involved in regulation of food intake, carbohydrate and lipid metabolism and hence energy hemostasis [8]. It also has multiple roles in the reproductive system as well as inflammatory and immune reactions [9]. Cardiotrophin-1 (CT-1) is a member of the IL- 6 superfamily like leptin and shares glycoprotein (gp) 130 as a receptor. CT-1 is also known as a key regulator of energy homeostasis [10].

Body mass index (BMI) and gender are the baseline characteristic factors affecting serum leptin concentrations. Leptin levels in women are two to three times higher than men with the same BMI [11]. This situation can be explained by a higher ratio of adipose tissue, which is the main production tissue of leptin. But there are inconsistent results on the relationship between $\mathrm{CT}-1$ and BMI. Obese subjects showed significantly lower CT-1 levels, and these levels were inversely correlated with $\mathrm{BMI}$ in a cohort of 81 patients with a high prevalence of diabetes, hypertension and metabolic syndrome [12]. In Rendo-Urteaga et al.' study, individuals with higher BMI had lower CT-1 levels [13]. In a study of overweight and normal weight adolescent subjects, CT-1 levels did not differ and BMI did not correlate with CT-1 [14]. In another study, compared with normal weight subjects, increased CT-1 levels were observed in obese patients [15]. In many studies the role of leptin in the pathogenesis of CVDs is reported, too [16-18]. Leptin exerts many atherogenic effects that are involved in atherosclerosis pathophysiology. Leptin provokes hypertension by promoting oxidative stress in endothelial cells. Leptin decreases vascular expandability by stimulating arterial smooth muscle cell migration and proliferation $[19,20]$. It has been noted that for coronary heart disease, leptin is a novel risk factor [21]. However, the precise role of leptin has not been determined in the cardiovascular system, yet. As an originally identified factor, CT-1 could induce hypertrophy on cardiac myocytes in vitro [10], and also CT-1 has been identified as an active inducer of cardiomyopathies, cardiac hypertrophy, hypertension, atherosclerosis, valvular heart disease, congestive heart failure, and acute coronary syndrome [12-15, 22] The aim of the present study is to evaluate the relationship between two risk factors for CVDs (CT-1 and leptin) and OSAS, and also the severity of the disease.

\section{Material and methods}

\section{Study groups}

Serum CT-1 and leptin concentrations were determined in patients who were suspected of sleep apnea and referred to the sleep unit at the Chest Diseases Clinic of Kayseri Education and Research Hospital, Turkey. The local ethics committee approved this study. All patients gave their written informed consent. Fifty newly diagnosed OSAS patients with excessive daytime sleepiness and thirty nonapneic controls participated in this study. In order not to effect leptin levels, we excluded the gender factor. So we chose only male participants for the patient and control groups in our study. Obstructive apnea was defined as a cessation of airflow for at least $10 \mathrm{~s}$ [23]. Hypopnea was defined as an arousal, or a reduction in respiratory airflow of $\geq 30 \%$ for at least $10 \mathrm{~s}$ plus an oxygen desaturation of $>4 \%$, or a reduction of airflow of $\geq 50 \%$ for at least $10 \mathrm{~s}$ plus an oxygen desaturation of $>3 \%$. AHI was defined as the mean number of hypopneic episodes plus apneic episodes per hour of sleep. According to their AHI, included subjects were grouped as mild/moderate (AHI between 5 and 29.9) and severe (AHI > 30). The control group consisted of patients with $\mathrm{AHI}<5$. $\mathrm{AHI}$ groups of patients are shown in Table I. Patient groups were created as group A (mild/moderate OSAS) and group B (severe OSAS), according to their AHI. There were 11 patients in group A (22\%) and 39 patients in group B (78\%) (Table II). Upper airway resistance, lung disease, heart failure, cardiovascular disease, cerebrovascular disease, chronic renal failure, systemic steroid therapy, hormone replacement therapy and central sleep apnea were the exclusion criteria in this study. By a standardized questionnaire, the participants' data (age, history of chronic and/or metabolic diseases, drugs, habits and cigarette smoking status) 
Table I. Comparison of baseline characteristics and findings between OSAS patients and control group

\begin{tabular}{|c|c|c|c|}
\hline Baseline characteristics and findings & OSAS & Control & $P$-value \\
\hline N & 50 & 30 & - \\
\hline \multicolumn{4}{|l|}{ Gender: } \\
\hline Male & 50 & 30 & - \\
\hline Age [years] & $47.40 \pm 13.30^{a}$ & $43.23 \pm 10.50^{\mathrm{a}}$ & NS \\
\hline \multicolumn{4}{|l|}{ AHI groups: } \\
\hline $0-4.9$ & - & 30 & $<0.001$ \\
\hline $5-14.9$ & $1(2 \%)$ & - & - \\
\hline $15-29.9$ & $10(20 \%)$ & - & - \\
\hline$>30$ & $39(78 \%)$ & - & - \\
\hline BMI $\left[\mathrm{kg} / \mathrm{m}^{2}\right]:$ & & & NS \\
\hline$<24.9$ & $5(10 \%)$ & $3(10 \%)$ & \\
\hline $25-29.9$ & $20(40 \%)$ & $12(40 \%)$ & - \\
\hline $30-34.9$ & $15(30 \%)$ & $9(30 \%)$ & - \\
\hline $35-39.9$ & $5(10 \%)$ & $3(10 \%)$ & - \\
\hline$>40$ & $5(10 \%)$ & $3(10 \%)$ & - \\
\hline Cigarette smoking (current) & 50 & 30 & - \\
\hline \multicolumn{4}{|l|}{ Comorbidity: } \\
\hline HT & $1(2 \%)$ & $0(0 \%)$ & - \\
\hline Diabetes mellitus & $4(8 \%)$ & $3(10 \%)$ & \\
\hline Triglycerides [mg/dl] & $175.50^{\dagger}(130.75 ; 238.75)$ & $166.0^{\dagger}(105.75 ; 192.75)$ & NS \\
\hline Total cholesterol [mg/dl] & $205.0^{\dagger}(178.0 ; 237.0)$ & $195.0^{\dagger}(177.5 ; 217.5)$ & NS \\
\hline LDL cholesterol [mg/dl] & $128.34 \pm 31.72^{\mathrm{a}}$ & $122.96 \pm 32.12^{\mathrm{a}}$ & NS \\
\hline HDL cholesterol [mg/dl] & $42.62 \pm 7.29^{\mathrm{a}}$ & $43.20 \pm 6.33^{a}$ & NS \\
\hline Cardiotrophin-1 [pg/ml] & $19.47^{\dagger}(10.34 ; 35.52)$ & $8.23^{\dagger}(4.16 ; 22.37)$ & $<0.0001$ \\
\hline Leptin [ng/ml] & $2.07^{\dagger}(1.33 ; 4.59)$ & $1.29^{\dagger}(0.7 ; 1.94)$ & $<0.0001$ \\
\hline
\end{tabular}

${ }^{\dagger}$ Mann-Whitney test; data are median and interquartile range (25\%; 75\%). ${ }^{a}$ t-test; data are mean \pm SD. NS - non-significant, OSAS obstructive sleep apnea syndrome, $\mathrm{AHI}$ - apnea-hypopnea index, $\mathrm{BMI}$ - body mass index.

Table II. Cardiotrophin-1 and leptin levels by OSAS classification of the patients

\begin{tabular}{|lccc|}
\hline Parameter & Group A & Group B & $P$-value \\
\hline OSAS severity & Mild/moderate & Severe & - \\
\hline Number of patients & 11 & 39 & - \\
\hline Cardiotrophin-1 $[\mathrm{pg} / \mathrm{ml}]$ & $10.34^{\dagger}(9.6 ; 19.72)$ & $21.70^{\dagger}(12.81 ; 49.61)$ & 0.013 \\
\hline Leptin $[\mathrm{ng} / \mathrm{ml}]$ & $2.07^{\dagger}(1.33 ; 2.89)$ & $1.92^{\dagger}(1.33 ; 5.85)$ & $\mathrm{NS}$ \\
\hline
\end{tabular}

${ }^{\dagger}$ Mann-Whitney test. Data are median and interquartile range (25\%; 75\%). NS - non-significant, OSAS - obstructive sleep apnea syndrome.

were recorded. Body mass index was calculated for each participant before the sleep study. All of the participants were current smokers. Four of the patients and three of the control subjects were diabetic. Only one participant in the patient group was hypertensive (Table I). Venous blood samples were drawn from all participants in the early hours on the morning after performing PSG.

\section{Polysomnographic evaluation}

Full night diagnostic PSG was applied to all subjects in a single-bedded room, in the Center for 
Snoring and Sleep Disorders of the Chest Diseases Clinic of Kayseri Education and Research Hospital. During spontaneous sleep with a supervising technician, patients' electrocardiogram (ECG), electroencephalogram (EEG), right-left electrooculogram (EOG), submental and right/left tibial electromyogram (EMG), body position parameters, thoracic and abdominal respiratory movements, blood oxygen saturation with pulse oximetry and of course their nasal air flow were recorded throughout the night. Participants were divided into four groups according to their AHI severity (Table I). All patients slept at least $6 \mathrm{~h}$, and at least $50 \%$ of their sleeping time was recorded.

\section{Lipid profile}

Fasting serum cholesterol $(\mathrm{mg} / \mathrm{dl})$, triglyceride $(\mathrm{mg} / \mathrm{dl})$, LDL cholesterol $(\mathrm{mg} / \mathrm{dl})$ and HDL cholesterol (mg/dl) levels were measured on an AU 2700 instrument (Beckman Coulter, California, USA). There was no statistically significant difference between the two groups in terms of lipid profile (Table I).

\section{Cardiotrophin-1 and leptin measurement}

On the morning after performing PSG, venous blood samples were drawn. They were centrifuged for $10 \mathrm{~min}$ at $2000 \mathrm{rpm}$, and serum was separated and stored at $-80^{\circ} \mathrm{C}$ until the assays were determined. Serum CT-1 and leptin concentrations were measured by commercial enzyme linked immunosorbent assay kits (USCN Life Science Inc. and DIAsource ImmunoAssays S.A., respectively) based on the protocol provided by the manufacturers. The minimum detectable concentrations of CT-1 and leptin were $6.3 \mathrm{pg} / \mathrm{ml}$ and $0.04 \mathrm{ng} / \mathrm{ml}$, respectively. The intra-assay and inter-assay coefficient of variation, respectively, were $<10 \%$ and $<10 \%$ for leptin and $<10 \%$ and $<12 \%$ for CT -1 .

\section{Statistical analysis}

Statistical analysis was carried out using SPSS software version 23.0 for Windows (SPSS Inc., USA). The results of groups with normal distribution are presented as mean $\pm S D$, and the median was used to present results that showed abnormal distribution. Categorical variables were pre- sented as percentages. To determine significant differences between the groups, the $t$-test was used for data with normal distribution and the Mann-Whitney $U$ test was used for data with non-normal distribution. To determine the relationship between the variables, for each group, Spearman's correlation coefficient was used. A receiver operating characteristic (ROC) analysis was performed to determine the best cut-off value. $P$-values $<0.05$ were accepted as statistically significant.

\section{Results}

Patients' and control subjects' baseline characteristics and clinical findings are listed in Table I. Fifty male OSAS patients with a mean age of $47.40 \pm 13.30$ years were enrolled in our study. The control group included 30 healthy male subjects, in terms of body mass index similar to the patient group, with a mean age of $43.23 \pm 10.50$ years and without apneas during sleep or pulmonary diseases. No statistically significant difference in age was found between the two groups $(p>0.05)$. The median CT-1 level of the patients was statistically significantly higher than the median CT-1 level of the control group (19.47 pg/ml and $8.23 \mathrm{pg} / \mathrm{ml}$ respectively) ( $p<0.001)$. The patients' serum leptin levels were found to be significantly higher than the levels of the control group. The difference between the median values of serum leptin in these two groups $(2.07 \mathrm{ng} / \mathrm{ml}$ and $1.29 \mathrm{ng} / \mathrm{ml}$ respectively) was statistically significant $(p<0.001)$. The serum median level of CT-1 was statistically significantly higher in group $B$ than the level in group $A$ (21.70 pg/ml and $10.34 \mathrm{pg} / \mathrm{ml}$ respectively) but leptin levels were not different between the two OSAS groups (Table II). In order to evaluate the correlation between patients' lipid profile and CT-1 and/or leptin levels, we performed Spearman's correlation analysis. Although there was no correlation between patients' leptin and lipid profile parameters, their CT-1 levels were positively correlated with HDL levels ( $p=0.02$ ) (Table III). But CT-1 concentrations were not associated with triglyceride, cholesterol or LDL cholesterol levels. According to a ROC analysis of serum CT-1 and leptin, the ROC rederived cut-off level for CT-1 was $14.165 \mathrm{pg} / \mathrm{ml}$ (AUC: 0.736; 95\% confidence inter-

Table III. $P$ and $r$ values of the Spearman's correlation analyses between cardiotrophin-1, leptin and lipoprotein levels in OSAS patients $(n=50)$

\begin{tabular}{|lcccc|}
\hline Parameter & Triglycerides & Total cholesterol & LDL cholesterol & HDL cholesterol \\
\hline Cardiotrophin-1 & $p=0.592$ & $p=0.116$ & $p=0.340$ & $p=0.022^{*}$ \\
& $r=0.078$ & $r=0.225$ & $r=0.138$ & $r=0.323$ \\
\hline Leptin & $p=0.622$ & $p=0.734$ & $p=0.955$ & $p=0.332$ \\
& $r=-0.071$ & $r=-0.049$ & $r=0.008$ & $r=-0.140$ \\
\hline
\end{tabular}

${ }^{\star} P<0.05$. OSAS - obstructive sleep apnea syndrome. 
val $(\mathrm{Cl}): 0.619-0.854,66 \%$ sensitivity, $70 \%$ specificity, $p<0.001)$ and the cut-off level for leptin was $1.74 \mathrm{ng} / \mathrm{ml}$ (AUC: $0.743 ; 95 \% \mathrm{Cl}: 0.636-0.850,62 \%$ sensitivity, $63 \%$ specificity, $p<0.001$ ) (Figure 1 ).

\section{Discussion}

The OSAS is a respiratory disorder characterized by recurrent episodes of apnea and hypopnea that results in the reduction or cessation of airflow [24]. In the literature there is evidence that overweight acts directly upon the pathophysiology of the narrowing of the upper airways and involvement of the oropharyngeal muscles, so obesity is a risk factor for OSAS [25]. The OSAS has a prevalence of approximately $40 \%$ in obese individuals, and about $70 \%$ of OSAS patients are diagnosed as obese [8]. In our study $50 \%$ of OSAS patients had $\mathrm{BMI}>29.9 \mathrm{~kg} / \mathrm{m}^{2}$.

The OSAS has been reported to induce CVDs. The CT-1 and leptin have been found to play a significant role in obesity, and obesity clearly has an important role in OSAS. Leptin and CT-1 are both members of the IL-6 superfamily and described as risk factors of CVDs. In many studies the role of leptin in the pathogenesis of CVDs is reported [16-18]. But, in the cardiovascular system, the precise role of leptin has not been determined yet. The CT-1 was identified as an inducer of hypertrophy on cardiac myocytes in vitro. Its active inducer effects on cardiomyopathies, valvular heart disease, congestive heart failure, atherosclerosis, hypertension, cardiac hypertrophy and acute coronary syndrome have also been described [10, 12$15,21]$. Inflammation is an another potential risk factor in OSAS. Hypoxia leads to oxidative stress, activates the systemic inflammatory response, and increases proinflammatory cytokines and antioxidant activity. Inflammatory cytokines were found to be associated with OSAS [26]. Leptin and CT-1 are significant markers of inflammation as an indicator of a systemic inflammatory response. Inflammation is known to be associated with CVDs and elevated concentrations of leptin and CT-1 as an inflammatory agents were measured in our patients with OSAS. In the literature there is only one study evaluating CT-1 and IL- 6 levels in OSAS patients [27]. In this study Kurt et al. found no significant difference in the plasma levels of CT- 1 and IL- 6 between the OSAS group and the controls. So to the best of our knowledge, this is the first report describing a significant difference in the serum levels of CT-1 between OSAS patients and controls, and a statistically significant difference between two OSAS groups. Our study showed that serum CT-1 and leptin levels were higher in OSAS patients compared with healthy controls. Serum CT-1 levels in severe OSAS patients were significantly higher than those in mild/moderate

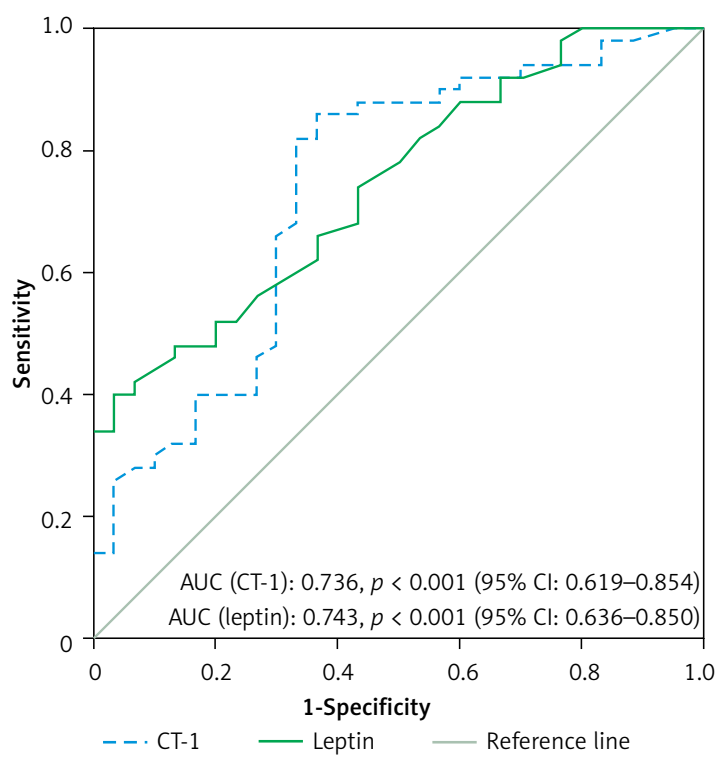

Figure 1. Receiver operating characteristics (ROC) curve analysis for cut-off value of $14.165 \mathrm{pg} / \mathrm{ml}$ and $1.74 \mathrm{ng} / \mathrm{ml}$ for CT-1 and leptin, respectively $A \cup C$ - area under the curve, $\mathrm{Cl}$-confidence interval.

OSAS patients (Table II) and CT-1 levels were positively correlated with patients' HDL levels.

In conclusion, our findings suggest that elevated serum CT-1 levels are associated with the presence and severity of OSAS. In our study we found that OSAS increases the serum concentrations of CT-1 and leptin. In our study cut-off values of serum CT-1 and leptin for OSAS were $14.165 \mathrm{pg} / \mathrm{ml}$ and $1.74 \mathrm{ng} / \mathrm{ml}$, respectively (Figure 1). So, OSAS patients who have values above these concentrations should be evaluated in terms of CVD risk. The CT-1 and leptin can be used as early markers in OSAS patients with asymptomatic/or without known CVDs.

Our relatively small sample size and the evaluation of only two adipokines were the limitations of our study. Further studies with larger sample size and long-term follow-up after CPAP treatment are warranted to elucidate the predictive value of CT-1 and leptin in OSAS patients.

\section{Conflict of interest}

The authors declare no conflict of interest.

\section{References}

1. Young T, Peppard PE, Gottlieb DJ. Epidemiology of obstructive sleep apnea: a population health perspective. Am J Respir Crit Care Med 2002; 165: 1217-39.

2. Young T, Palta M, Dempsey J, Skatrud J, Weber S, Badr S. The occurrence of sleep disordered breathing among middle-aged adults. N Engl J Med 1993; 328: 1230-5.

3. Shahar E, Whitney CW, Redline S, et al. Sleep-disordered breathing and cardiovascular disease: cross-sectional results of the sleep heart health study. Am J Respir Crit Care Med 2001; 163: 19-25. 
4. Malhotra A, White DP. Obstructive sleep apnea. Lancet 2002; 360: 237-45.

5. Somers VK, White DP, Amin R, et al. American Heart Association Council for High Blood Pressure Research Professional Education Committee, Council on Clinical Cardiology; American Heart Association Stroke Council; American Heart Association Council on Cardiovascular Nursing; American College of Cardiology Foundation. Sleep apnea and cardiovascular disease: an American Heart Association/american College Of Cardiology Foundation Scientific Statement from the American Heart Association Council for High Blood Pressure Research Professional Education Committee, Council on Clinical Cardiology, Stroke Council, and Council On Cardiovascular Nursing. In collaboration with the National Heart, Lung, and Blood Institute National Center on Sleep Disorders Research (National Institutes of Health). Circulation 2008; 118: 1080-111.

6. Destors M, Tamisier R, Baguet JP, Levy P, Pepin JL. Cardiovascular morbidity associated with obstructive sleep apnea syndrome. Rev Mal Respir 2014; 31: 375-85.

7. Zamarrón C1, Riveiro A, Gude F. Circulating levels of vascular endothelial markers in obstructive sleep apnoea syndrome. Effects of nasal continuous positive airway pressure. Arch Med Sci 2011; 7: 1023-8.

8. Tsutamoto T, Asai S, Tanaka T, et al. Plasma level of cardiotrophin-1 as a prognostic predictor in patients with chronic heart failure. Eur J Heart Fail 2007; 9: 1032-7.

9. González A, López B, Martín-Raymondi D, et al. Usefulness of plasma cardiotrophin-1 in assessment of left ventricular hypertrophy regression in hypertensive patients. J Hypertens 2005; 23: 2297-304.

10. Moreno-Aliaga MJ, Pérez-Echarri N, Marcos-Gómez B, et al. Cardiotrophin-1 is a key regulator of glucose and lipid metabolism. Cell Metab 2011; 14: 242-53.

11. Ostlund RE, Yang JW, Klein S, Gingerich R. Relation between plasma leptin concentration and body fat, gender, diet, age and metabolic covariates. J Clin Endocrinol Metab 1996; 81: 3909-13.

12. Vespasiani-Gentilucci U, De Vincentis A, Argemi J, et al. Cardiotrophin-1 is not associated with carotid or coronary disease and is inversely associated with obesity in patients undergoing coronary angiography. Arch Med Sci 2013; 9: 635-9.

13. Rendo-Urteaga T, García-Calzón S, Martínez-Ansó E, et al. Decreased cardiotrophin-1 levels are associated with a lower risk of developing the metabolic syndrome in overweight/obese children after a weight loss program. Metabolism 2013; 62: 1429-36.

14. Jung C, Fritzenwanger M, Figulla HR. Cardiotrophin-1 in adolescents: impact of obesity and blood pressure. Hypertension 2008; 52: e6.

15. Natal C, Fortuño MA, Restituto P, et al. Cardiotrophin-1 is expressed in adipose tissue and upregulated in the metabolic syndrome. Am J Physiol Endocrinol Metab 2008; 294: 52-60.

16. López B, Castellano JM, González A, Barba J, Díez J. Association of increased plasma cardiotrophin-1 with inappropriate left ventricular mass in essential hypertension. Hypertension 2007; 50: 977-83.

17. Talwar S, Downie PF, Squire IB, Davies JE, Barnett DB, Ng LL. Plasma N-terminal pro BNP and cardiotrophin-1 are elevated in aortic stenosis. Eur J Heart Fail 2001; 3: 15-91.

18. Talwar S, Squire IB, Downie PF, Davies JE, Ng LL. Plasma $\mathrm{N}$ terminal pro-brain natriuretic peptide and cardiotrophin 1 are raised in unstable angina. Heart 2000; 84 421-4.
19. Zhang Y, Proenca R, Maffei M, Barone M, Leopold L, Friedman JM. Positional cloning of the mouse obese gene and its human homolog. Nature 1994; 372: 425-32.

20. Margetic S, Gazzola C, Pegg GG, Hill RA. Leptin: a review of its peripheral actions and interactions. Int J Obes 2002; 26: 1407-33.

21. Wallace AM, McMahon AD, Packard CJ, et al. Plasma leptin and the risk of cardiovascular disease in the west of Scotland coronary prevention study (WOSCOPS). Circulation 2001; 104: 3052-6.

22. Pennica D, King KL, Shaw KJ, et al. Expression cloning of cardiotrophin 1, a cytokine that induces cardiac myocyte hypertrophy. Proc Natl Acad Sci USA 1995; 92: 1142-6.

23. Iber C, Ancoli-Israel S, Chesson A, Quan SF. American Academy of Sleep Medicine. The AASM manual for the scoring of sleep and associated events: rules, terminology and technical specifications. American Academy of Sleep Medicine, Westchester, IL, 2007.

24. Modena DAO, Cazzo E, Cândido EC, et al. Obstructive sleep apnea syndrome among obese individuals: a cross-sectional study. Rev Assoc Med Bras (1992) 2017; 63: 862-8.

25. Busetto L, Enzi G, Inelmen EM, et al. Obstructive sleep apnea syndrome in morbid obesity: effects of intragastric balloon. Chest 2005; 128: 618-23.

26. Lurie A. Inflammation, oxidative stress, and procoagulant and thrombotic activity in adults with obstructive sleep apnea. Adv Cardiol 2011; 46: 43-66.

27. Kurt OK Tosun M, Talay F. Serum cardiotrophin-1 and IL-6 levels in patients with obstructive sleep apnea syndrome. Inflammation 2013; 36: 1344-7. 